\title{
Azimuthally symmetric pseudosurface and helicon wave propagation in an inductively coupled plasma at low magnetic field
}

\author{
T. Lho, N. Hershkowitz, J. Miller, ${ }^{\text {a) }}$ W. Steer, and G. H. Kim ${ }^{\text {b) }}$ \\ Department of Engineering Physics, University of Wisconsin, 1500 Engineering Drive, Madison, \\ Wisconsin 53705
}

(Received 10 March 1998; accepted 14 May 1998)

\begin{abstract}
The mode transition from a capacitively coupled mode ( $E$ mode) to an inductively coupled mode $(H$ mode) was observed in an inductive Ar plasma source by applying an axially uniform low $B$ field. The applied fundamental rf was $13.56 \mathrm{MHz}$ and many harmonic components were observed. A beat and standing wave patterns of azimuthally symmetric $(m=0$ mode $)$ first and second harmonic pseudosurfaces and helicon waves were measured at various densities $\left(n \sim 9.0 \times 10^{10}-2.2\right.$ $\times 10^{11} \mathrm{~cm}^{-3}$ ) and $B$ fields $(12-28 \mathrm{G})$. Wave propagation mode changes, from pseudosurface to helicon waves and from helicon to pseudosurface waves, were observed at critical conditions, $\omega_{c} / \omega>3.0$ and $n \sim 2.2 \times 10^{11} \mathrm{~cm}^{-3}$. (C) 1998 American Institute of Physics.
\end{abstract}

[S1070-664X(98)02708-6]

\section{INTRODUCTION}

Inductively coupled plasma (ICP) sources are becoming common worldwide in plasma-aided manufacturing devices. They are used over a very wide range of operating pressure from atmosphere, e.g., plasma torches, to milliTorr, e.g., etching devices for semiconductor manufacturing. ${ }^{1,2}$ Early investigations of inductive sources demonstrated the mode transition from the electrostatically coupled mode ( $E$ mode) to the electromagnetically coupled mode ( $H$ mode). The $E$ mode is characterized by a low density, faint glow discharge at low input power. With increasing input power, the faint glow discharge suddenly becomes a high density, luminous discharge at a certain critical input power. MacKinnon ${ }^{3}$ reported the first explanation on this mode transition. At low input power the effect of the electrostatic $E$ field $\left(E_{s}\right)$ inside the chamber is much greater than that of the induced $E$ field $\left(E_{m}\right)$ and is responsible for sustaining the low density, faint glow discharge. By increasing the rf input power, $E_{m}$ becomes dominant and a sudden mode transition to the $H$ mode is observed. In the $H$ mode, the $E$ field induced by the antenna deposits energy within the skin depth and forms a luminescent localized high density plasma near the antenna. Hopwood et al. ${ }^{4}$ reported that it is possible to sustain the $H$ mode by an induced $E$ field strength of approximately $1-2 \mathrm{~V} \mathrm{~cm}^{-1}$. A mode transition is also observed in helicon plasma sources, which show a three-step mode transition, that is from $E$ mode to $H$ mode and finally to helicon mode. ${ }^{5}$

Smullin et al. ${ }^{6}$ recognized that when the phase velocity of an electromagnetic wave in a rectangular metallic wave guide filled with plasma with constant $B$ field satisfies $v_{\mathrm{ph}}$ $(=\omega / k) \ll c$, the wave can be treated by the electrostatic approximation. The existence of this mode was observed inde-

a)Present address: TwinStar Semiconductor LLC, 500 West Renner Rd., Richardson, TX 75080.

${ }^{b}$ Present address: Department of Physics, University of Hanyang-Ansan, Guynggi-Do, Ansan-Si, Korea. pendently by Trivelpiece and Gould. ${ }^{7}$ They measured azimuthally symmetric electromagnetic wave propagation in a cylindrical plasma column with an axially uniform $B$ field and found a dispersion relation of space charge waves, now known as Trivelpiece-Gould waves or more widely as surface waves. They made the electrostatic approximation and explained transverse magnetic (TM) wave propagation by the formation of the electric fields $\left(E_{z}\right)$ at the boundary of the plasma. Zhelyazkov et al. ${ }^{8}$ classified the guided electrostatic waves in more detail according to several ranges of $B$ field and density. In the case of overly dense plasma $\left(\omega_{p}\right.$ $>\omega)$, surface waves can sustain the plasma at weak $B$ field $\left(\omega_{c}<\omega\right)$ and the wave field is maximum at the boundary of the plasma. Meanwhile, pseudosurface waves have fields that are maximum at the center of the plasma for strong $B$ field $\left(\omega_{c}>\omega\right)$. In the limit of infinite $B$ field, all waves become TM modes, whatever the mode number is, and can be identified as a pure pseudosurface wave or volume waves. ${ }^{9}$ Benova et al. ${ }^{10}$ recently calculated the three-dimensional surface and pseudosurface waves structure numerically for finite $B$ field. They used an electromagnetic model consisting of a dispersion relation and an energy balance equation. With the $B$ field, the plasma becomes an anisotropic medium, which results in a coupled wave field structure of transverse electric (TE) and transverse magnetic (TM) modes whose wave $E$ and $B$ fields can have $r, \theta, z$ components in cylindrical geometry.

Klozenberg et al. ${ }^{11}$ found an unexpected wave attenuation in a helicon wave study, for which the electrical resistivity term in the equations was not involved. They reported the surface current required at the boundary of the plasma needed to match the plasma wave field to the vacuum wave field. Chen ${ }^{12}$ identified that the surface current was associated with a pseudosurface wave. Chen predicted the existence of pseudosurface waves in a helicon wave device at low $B$ field and a wave propagation mode change between 


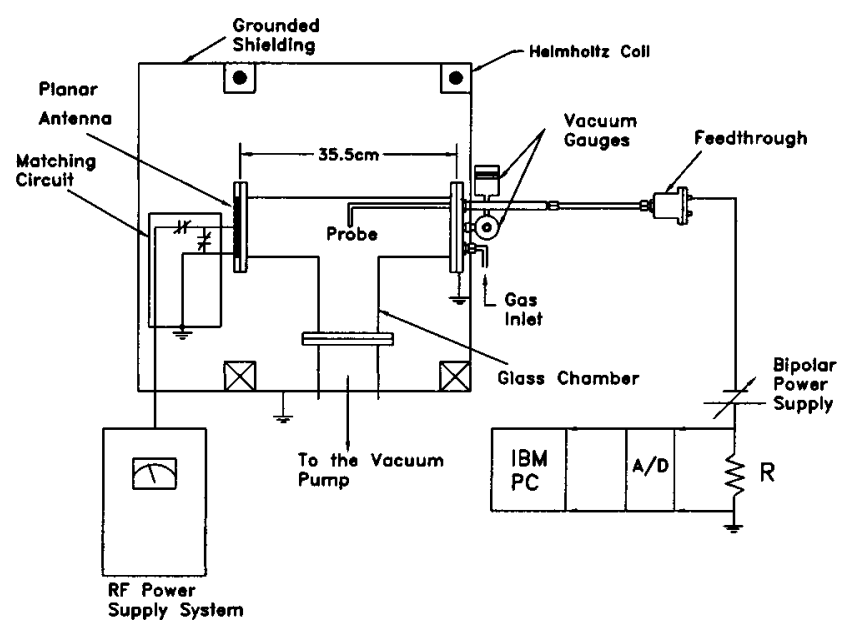

FIG. 1. Schematic drawing of the experimental setup.

pseudosurface waves and helicon waves under certain conditions.

In this paper, a plasma mode transition from an $E$ mode to an $H$ mode discharge is observed by applying an axially uniform external low $B$ field. Azimuthally symmetric ( $m$ $=0)$ pseudosurface and helicon wave propagation in $\mathrm{H}$ mode discharges with the $B$ field were measured and a propagation mode change between pseudosurface waves and helicon waves was observed at the conditions, $\omega_{c} / \omega>3.0$ and $n \sim 2.2 \times 10^{11} \mathrm{~cm}^{-3}$.

\section{EXPERIMENTAL SETUP}

The experimental system consisted of a chamber, matching box, power supply, and Helmholtz coil which produced an axially uniform $B$ field as seen in Fig. 1. Plasma parameters and induced wave $B$ field by the antenna were measured by a Langmuir probe and a $B$-dot probe.

A T-shaped glass tube was used as a chamber whose dimension are $L=35 \mathrm{~cm}$ and $D=10 \mathrm{~cm}$. The bottom part of the chamber was connected to the vacuum pump system. The system has a base pressure lower than $10^{-6}$ Torr. Argon gas was used. The operating pressure was 1-10 mTorr. An aluminum flange was mounted on the end of the chamber and served as the system ground. A $1 / 4$ in. quartz window was fixed by a Plexiglas ${ }^{\mathrm{TM}}$ flange upon which the rf antenna was mounted. A 1/8 in. copper tube was used for the planar fourturn spiral type antenna. The end of the antenna was connected to a matching circuit. The matching circuit consisted of two variable vacuum capacitors $(15-80 \mathrm{pF})$. Ground connections to the matching box made a common ground with the antenna ground, chamber ground, and ground shielding. A copper mesh enclosed the chamber, antenna, and matching box to shield the rf fields from the laboratory. A rf connecter was installed on the copper mesh and connected to the transmission line from the power supply. Two magnet coils, whose diameters were both $70 \mathrm{~cm}$, were separated by $35 \mathrm{~cm}$ to meet the Helmholtz condition, which is sufficient for an axially uniform $B$ field. Dimensions of the magnet coils were big enough to neglect the radial $B$-field variation in the chamber dimensions.
A cylindrical Langmuir probe $(D=0.5 \mathrm{~mm}, L$ $=2.5 \mathrm{~mm}$ ) was used to measure the ion density and electron temperature. To remove the rf coupled signal, an LC resonance filter was located $1 \mathrm{~cm}$ from the end of the tungsten (or graphite) probe tip. ${ }^{13}$ The LC resonance filter serially connected the several filter units to cut off not only the first harmonic $(f=13.56 \mathrm{MHz})$, but also higher harmonics up to the fifth harmonic. The data acquisition system consisted of an $\mathrm{AD}$ (analog-to-digital) converter and a PC which collected Langmuir probe data. A $B$-dot probe $(D \sim 3 \mathrm{~mm})$ was used to measure the wave $B$ field. To prevent capacitive coupling with the plasma, the $B$-dot probe was wrapped in a slotted stainless steel sheet for electrostatic shielding. The wave signal of each harmonic was distinguished by using a spectrum analyzer (Tektronix 7L14). The frequency spectrum was displayed on an $X-Y$ plotter.

\section{PSEUDOSURFACE AND HELICON WAVE DISPERSION RELATIONS}

Assuming stationary ions, the momentum equation of electron is

$$
m \frac{\partial \mathbf{v}}{\partial t}=-e\left(\mathbf{E}+\mathbf{v} \times \mathbf{B}_{0}\right)-m \nu \mathbf{v},
$$

where $m$ is the electron mass, $\mathbf{v}$ is the electron drift velocity, $\mathbf{E}$ is the electric field, $\mathbf{B}_{0}$ is the external $B$ field, and $\nu$ is the collisional frequency. Assuming axially uniform plasma density, the electron velocity

$$
\mathbf{v}=-\frac{\mathbf{j}}{n_{0} e}
$$

and collisional frequency

$$
\nu=\frac{n_{0} e^{2}}{m} \eta,
$$

where $n_{0}$ is plasma density and $\eta$ is the electrical resistivity. Assuming a plane wave solution, $B \propto e^{i(k z-\omega t)}$, and solving Eq. (1) for $\mathbf{E}$ in terms of $\mathbf{j}$ and $\eta, \mathbf{E}$ is

$$
\mathbf{E}=\frac{1}{n_{0} e} \mathbf{j} \times \mathbf{B}_{0}+\eta \mathbf{j}\left(1-\frac{i \omega}{\nu}\right) .
$$

If $\omega / k \ll c$, the quasistatic approximation can be employed and the displacement current term can be dropped in Maxwell's equations,

$$
\begin{aligned}
& \nabla \times \mathbf{E}=-\frac{\partial \mathbf{B}}{\partial t}, \\
& \nabla \times \mathbf{B}=\mu_{0} \mathbf{j} .
\end{aligned}
$$

Substituting Eqs. (4) and (6) into Eq. (5) and rewriting into a vector equation,

$$
(\omega+i \nu) \nabla \times \nabla \times \mathbf{B}-k \omega_{c} \boldsymbol{\nabla} \times \mathbf{B}+\left(\frac{\omega_{p}^{2} \omega}{c^{2}}\right) \mathbf{B}=0,
$$

where $\omega_{c}$ and $\omega_{p}$ are the cyclotron frequency and plasma frequency of electron, respectively. Equation (7) can be written: 


$$
\left(\beta_{s}-\nabla \times\right)\left(\beta_{h}-\nabla \times\right) \mathbf{B}=0,
$$

where $s, h$ denote the pseudosurface and helicon wave, respectively, and $\beta_{s, h}$ is the total wave number. The total wave number, $\beta_{s, h}$, satisfies

$$
\beta_{j}^{2}-\left(\frac{k \omega_{c}}{\omega+i v}\right) \beta_{j}+\left(\frac{\omega \omega_{p}^{2} / c^{2}}{\omega+i v}\right)=0,
$$

where $j=s, h$. This quadratic equation has two solutions, which are the pseudosurface and helicon wave dispersion relations,

$$
\beta_{j}=\frac{k_{j}}{2(\omega+i \nu)}\left[1 \pm \sqrt{1-\frac{4(\omega+i \nu)}{k_{j}^{2} \omega_{c}^{2}}\left(\frac{\omega \omega_{p}^{2}}{c^{2}}\right)}\right] .
$$

The plus $(+)$ sign gives the pseudosurface wave dispersion relation and the minus (-) sign gives the helicon wave dispersion relation. This dispersion relation was first derived by Klozenberg et al. ${ }^{11}$ Both waves satisfy the Helmholtz equation,

$$
\nabla^{2} B+\beta_{j}^{2} B=0 .
$$

Let us assume the plane wave solution for the $\theta$ and $z$ direction, $B \propto e^{i(m \theta+k z)}$. In a finite system, the Helmholtz equation becomes an eigenvalue problem and the solution of Eq. (11) for cylindrical geometry is

$$
B=B_{z 0} J_{m}(\operatorname{Tr}) e^{i(m \theta+k z)},
$$

where $J_{m}$ is the first kind of $m$ th order Bessel function. In this relation, the transverse wave number, $T$, is given by

$$
T=\frac{a_{m n}}{r},
$$

where $a_{m n}$ is the $n$th root of $m$ th order Bessel function and $r$ is the plasma radius. The transverse wave number is related to the total wave number, $\beta$, and the parallel wave number, $k$, by

$$
T_{j}^{2}=\beta_{j}^{2}-k_{j}^{2} .
$$

If the wave number, $k$, satisfies the following condition:

$$
2\left(\frac{\omega}{\omega_{c}}\right)\left(\frac{\omega_{p}}{c}\right)<k_{j}<\left[\frac{1}{\left(\omega_{c} / \omega\right)-1}\right]^{1 / 2}\left(\frac{\omega_{p}}{c}\right),
$$

solutions of Eq. (10) will be purely real values which means that two waves can propagate. Combining Eq. (15) with the pseudosurface wave solution of Eq. (10), the dispersion relation of the pseudosurface wave is

$$
k_{s}=T_{s}\left[\left(\frac{\omega_{c}}{\omega}\right)-1\right]^{-1 / 2} .
$$

This relation can be directly drived from the dispersion relation that Trivelpiece and Gould had driven if $\omega_{P} \gg \omega$. Making a similar manipulation, the dispersion relation of helicon waves is

$$
k_{h}^{2}=T_{h}^{2}\left(\frac{\sqrt{\left(1+4 k_{w}^{4}\right) / T_{h}-1}}{2}\right),
$$

where

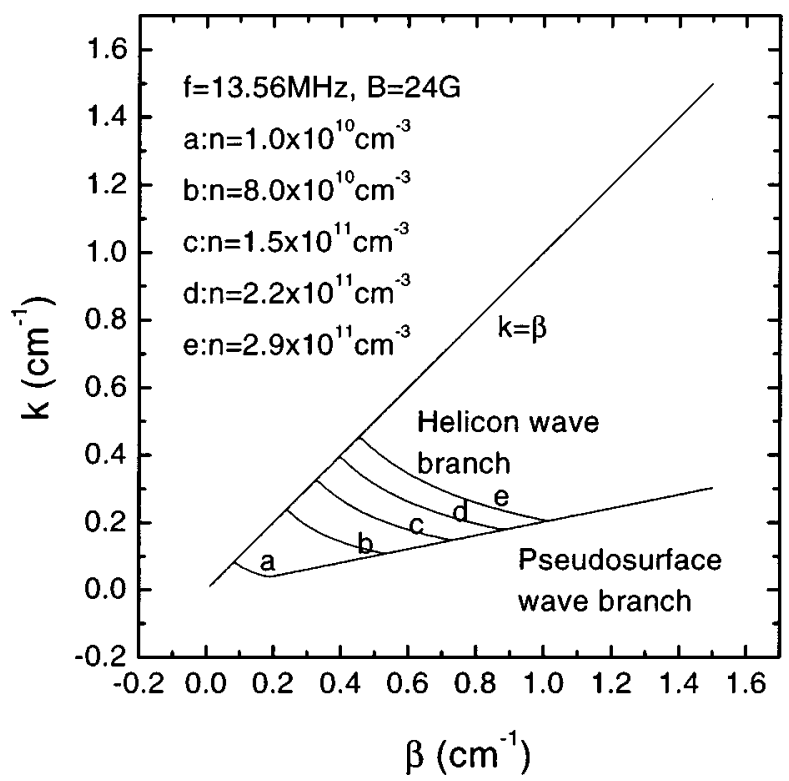

FIG. 2. Wave numbers of helicon and pseudosurface waves at $f$ $=13.56 \mathrm{MHz}, \omega_{c} / \omega \sim 5$ for various densities.

$$
k_{w}^{2}=\frac{\omega}{\omega_{c}}\left(\frac{\omega_{p}}{c}\right)^{2} .
$$

Assuming, $T_{h}^{2} \gg k_{h}^{2}$ or $4 k_{w}^{4} / T_{h}^{4} \gg 1$, Eq. (17) becomes

$$
k_{h} \sim \frac{\omega}{T_{h}} \frac{n_{0} \mu_{0} e}{B_{0}} \text {. }
$$

This is the well-known helicon dispersion relation. $T_{h}$ is also determined by Eq. (13). Note that this dispersion relation shows a density dependence, while the pseudosurface waves dispersion relation, Eq. (16), is independent of density if $\omega_{p} \gg \omega$. This point is a major difference between the two waves. Comparing the dispersion relations of the two waves, it is apparent that at high density the helicon waves become dominant (see Fig. 2).

\section{EXPERIMENTAL RESULTS}

The first stage of plasma generation in this experiment can be characterized as an $E$ mode with a low density, faint glow discharge. Plasma sustains the $E$ mode until the $B$ field reaches approximately $19 \mathrm{G}$ as seen in Fig. 3. The reflected power in the $E$ mode is about $7 \%-15 \%$ of the input power and the measured ion density is approximately $\sim 10^{9} \mathrm{~cm}^{-3}$. With increasing $B$ field, the plasma mode suddenly changes to the high density mode ( $H$ mode) near $B_{0}=19 \mathrm{G}$, showing almost $0 \%$ reflected power. The input power decreases to $40 \%$ of the input power present before the mode change. It appears the variation of reflection and input power comes from the variation of the matching condition by the changing of the plasma density. The sudden mode change near $B_{0}$ $=19 \mathrm{G}$ is consistent with an electron cyclotron resonance at the fourth harmonic. When the $B$ field is greater than $26 \mathrm{G}$, plasma returns to a low density, faint glow discharge which resembles the $E$ mode.

The plasma discharge mode returns to the high density mode when the $B$ field is decreased. As the $B$ field decreases 


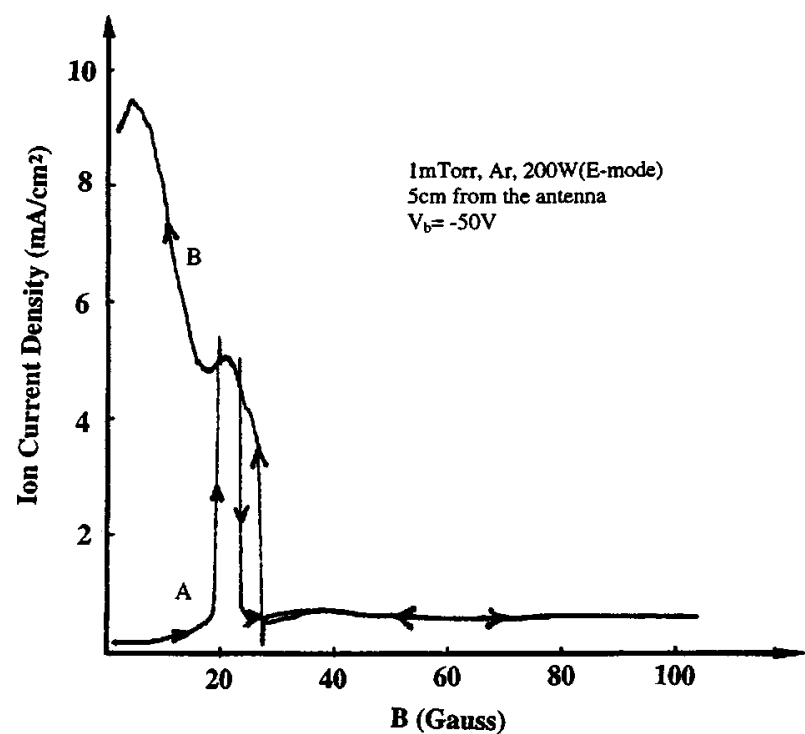

FIG. 3. Plasma density variation with $B$ field. The curve A for $B_{0}<20 \mathrm{G}$ corresponds to the $E$ mode, curve B corresponds to the $H$ mode. Once plasma changes to the $H$ mode near $B_{0}=19 \mathrm{G}$, it stays in the $H$ mode. Density decreases for curve B with increasing $B$ field is a result of the expansion of the plasma column axially. Its axially averaged density is approximately constant with $B$ field, while it becomes axially more uniform.

further, the plasma does not return to the low density mode. Instead, it sustains the high density mode as seen in curve B in Fig. 3. When the $B$ field is removed from the system, high density plasma is localized near the antenna and is characterized by the luminous discharge of the $H$ mode. This kind of irreversible process can qualitatively be explained by MacKinnon's description ${ }^{3}$ on the mode transition in inductively coupled plasma sources. What differentiates the present work from the previous observation on the mode transition is that the $B$ field causes the transition instead of power or pressure. Once the plasma jumps to the $H$ mode, it will sustain high density and move along the curve $\mathrm{B}$ with changing $B$ field. The electromagnetic field induced by the antenna deposits its energy within the skin depth and forms localized plasma near the antenna at $B_{0}=0 \mathrm{G}$. With the $B$ field the plasma becomes an anisotropic medium in which electromagnetic waves can propagate. Although curve B shows plasma density decreasing with the $B$ field, the plasma column expands along the $B$ field and becomes axially uniform. When the $B$ field is greater than $26 \mathrm{G}$, the plasma returns to the low density mode. With increasing $B$ field, the plasma uniformity is enhanced and changes the matching condition. When the $B$ field is over $26 \mathrm{G}$ in Fig. 3, the system is no longer well matched and cannot sustain the high density mode. If the $B$ field is less than $26 \mathrm{G}$, the plasma recovers the high density mode as seen in curve B. Hence, the discontinuity of the plasma density on curves $\mathrm{A}$ and $\mathrm{B}$ at about $B_{0}=26 \mathrm{G}$ comes from the effect of the $B$ field on system impedence matching. It was hard to get good impedence matching at low density $\left(n \leqslant 9.0 \times 10^{10} \mathrm{~cm}^{-3}\right)$ if the $B$ field was greater than $26 \mathrm{G}$. But at high density $(n \leqslant 2.2$ $\times 10^{11} \mathrm{~cm}^{-3}$ ) impedence matching was easily achieved over $30 \mathrm{G}$.

An axially averaged density is used for the wave disper-

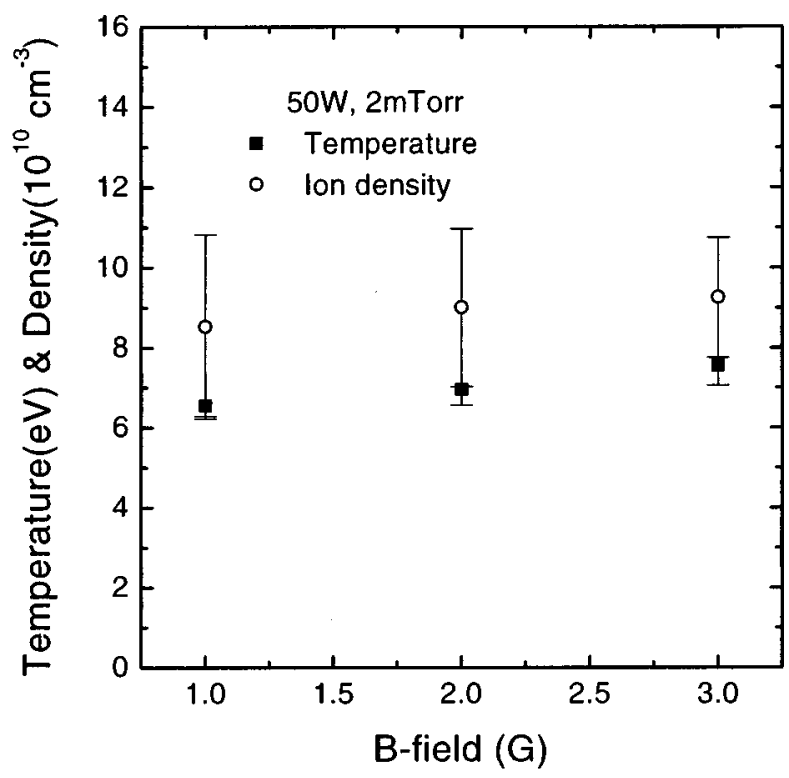

(a)

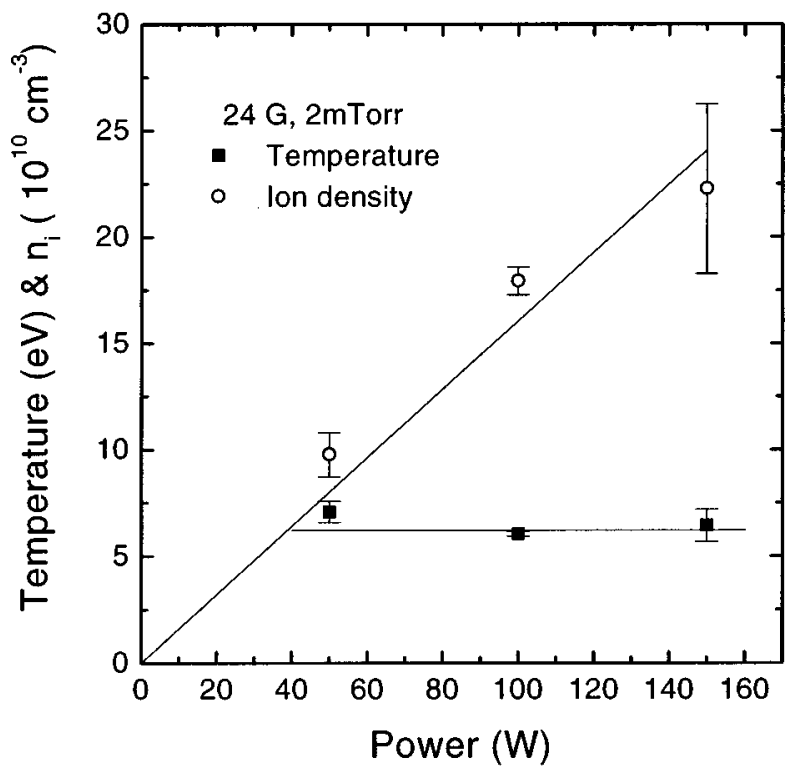

(b)

FIG. 4. Plasma density and temperature variations with power and $B$ field. (a) Plasma density and temperature in the $H$ mode are almost independent of the $B$ field. Error bars represent the axial density variation. (b) Plasma density is linearly increasing with power while temperature is almost constant.

sion relation even though the axial variation is not uniform. For density measurements, the matching condition has been changed, whenever the $B$ field is changed, to keep the reflected power under $8 \%$ of input power. The measured plasma density and temperature with power and $B$ field are shown in Fig. 4. The axially averaged density is approximately constant with the changing $B$ field. The presence of the $B$ field enhances the plasma uniformity as shown in Fig. 4(a). The temperature is almost independent of the $B$ field and produces an axially uniform distribution. Hence, the density and the temperature are essentially independent of the variation of $B$ field. The density increases linearly with 


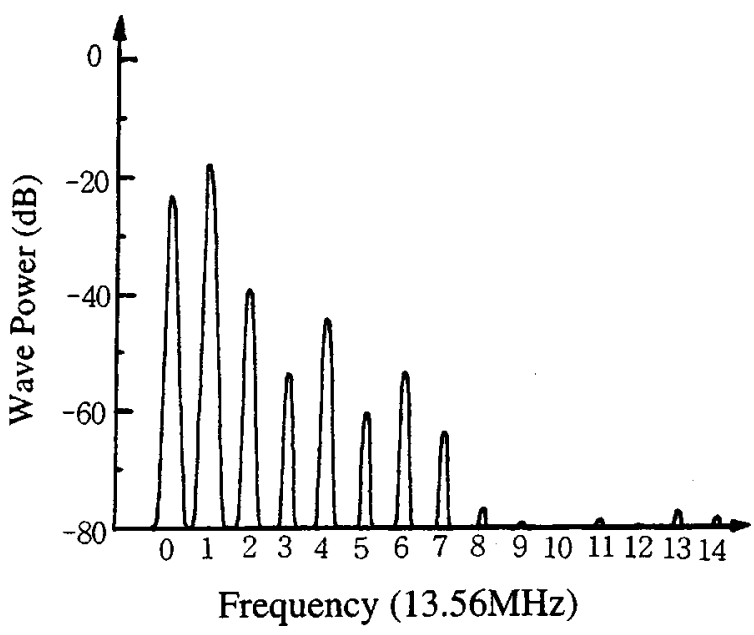

FIG. 5. Wave power spectrum. Experimental conditions are $B_{0}=16 \mathrm{G}, 100$ $\mathrm{W}$ input power, and 2 mTorr Ar plasma. $B$-dot probe measurements were performed $7 \mathrm{~cm}$ from the antenna.

power while the temperature shows a $\pm 1 \mathrm{eV}$ variation as seen in Fig. 4(b).

After the mode transition, both electrostatic $E$ fields $\left(E_{s}\right)$ and induced $E$ fields $\left(E_{m}\right)$ are coupled into the plasma. This extra $E_{s}$ may produce higher harmonics by nonlinear processes. The wave power spectrum is given in Fig. 5. Only harmonics which satisfy the requirement $\omega_{c}>\omega$ are investigated. This includes frequencies up to the fifth harmonic. The wave structure can be obtained from wave power spectra measured along the chamber. Generally, it is possible to obtain two types of wave patterns; one is a beat structure of two waves and the other is a standing wave pattern. In this experiment, both types of wave patterns were measured. The characteristics of beat wave structures in this experiment are that their minimum values in the interior of the chamber are close to zero. After a careful analysis, the wave patterns at low power $\left(n \leqslant 9.0 \times 10^{10} \mathrm{~cm}^{-3}\right)$ were identified as beat structures. In the case of the standing wave patterns, the minimum values are far from zero in the interior of the chamber. At high density and high $B$ field, the wave patterns can be treated as standing wave patterns. Because wave power was measured, for both beat and standing wave patterns, the distance from a minimum to the next minimum, or maximum to the next maximum, corresponds to half of the wavelength.

Clear wave patterns are observable at the first harmonic $(f=13.56 \mathrm{MHz})$ and the second harmonic $(f=27.12$ $\mathrm{MHz}$ ), but not at the other harmonics. The $B$ field associated with waves without an external $B$ field is seen to be an evanescent wave. With an external $B$ field, the pattern starts to show a beat wave when the external $B$ field is over $12 \mathrm{G}$. The measured beat and standing wave patterns at the first and second harmonics are shown in Figs. 6 and 7, respectively. As seen in Fig. 6(a), the wave pattern at low $B$ field $\left(\omega_{c} / \omega \sim 3.3\right)$ is independent of the plasma density. At high density $\left(n=2.2 \times 10^{11} \mathrm{~cm}^{-3}\right)$ and high $B$ field $\left(\omega_{c} / \omega\right.$ $\sim 5.0$ ), it becomes a short wavelength mode with a standing wave pattern as shown in Fig. 6(b). The wavelength at the

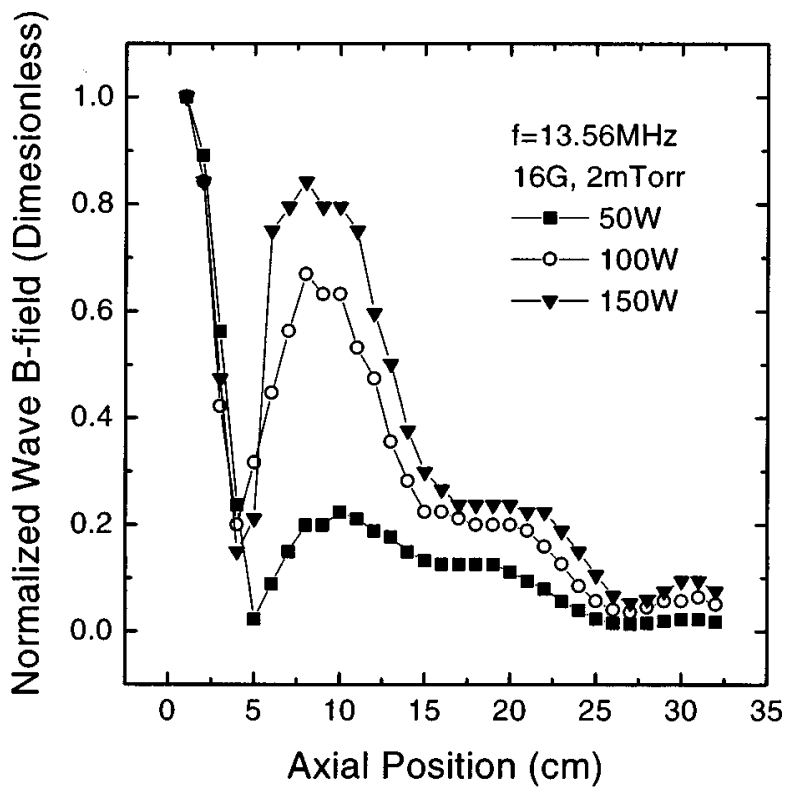

(a)

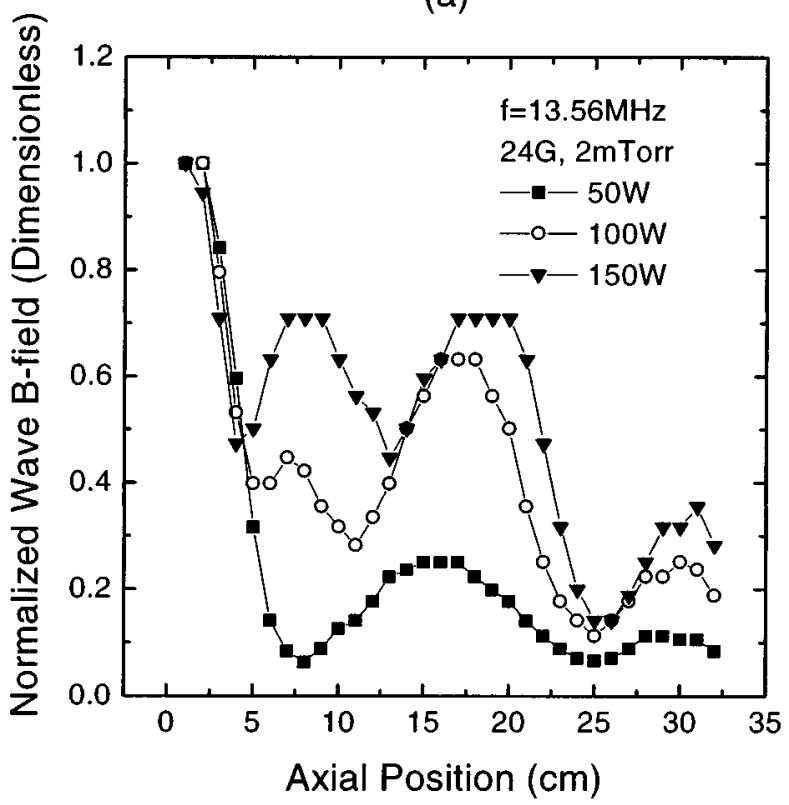

(b)

FIG. 6. (a) Beat wave patterns of the first harmonic at $\omega_{c} / \omega \sim 3.3$. The wavelengths are not changed with the input power proportional to the plasma density. (b) Beat and standing wave patterns at $\omega_{c} / \omega \sim 5$; the long wavelength beat pattern at low power is changed to a short wavelength standing wave pattern at an input power of $150 \mathrm{~W}$, which corresponds to the average density, $n \sim 2.2 \times 10^{11} \mathrm{~cm}^{-3}$.

second harmonic shows a density dependence at $\omega_{c} / \omega$ $\sim 1.65$ and a density independence at $\omega_{c} / \omega \sim 2.5$ as shown in Figs. 7(a) and 7(b). This density dependence of wavelength is one major difference between pseudosurface waves and helicon waves as given in Eqs. (16) and (18). From these results the existence of two waves can be qualitatively explained.

Usually, in helicon wave experiments with a Nagoya type III or Boswell type antennas, ${ }^{14} k$ and $T$ are determined by the antenna dimensions and are assumed constant. On the other hand the $m=0$ type antenna in this experiment does 


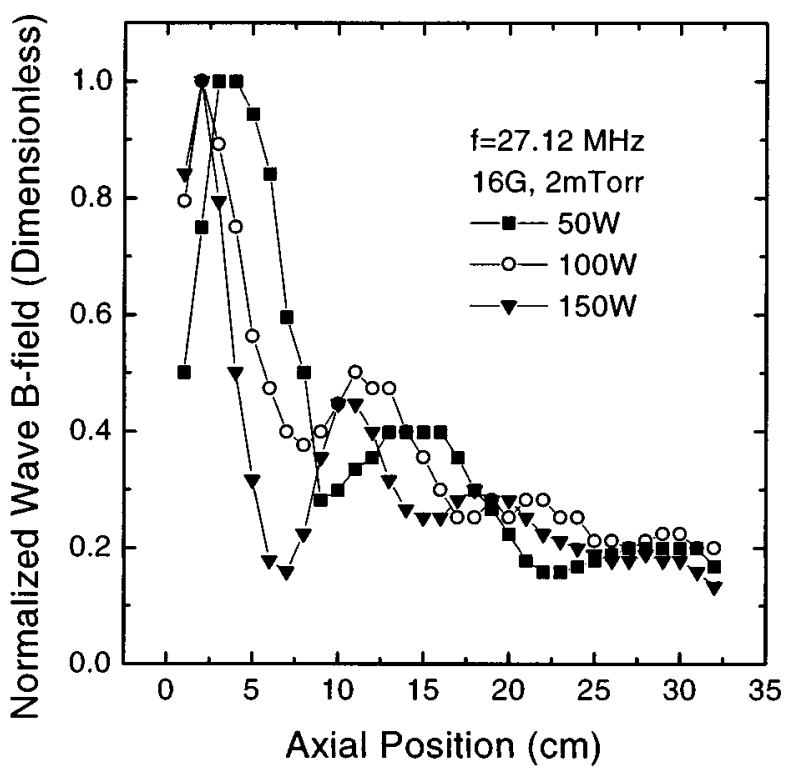

(a)

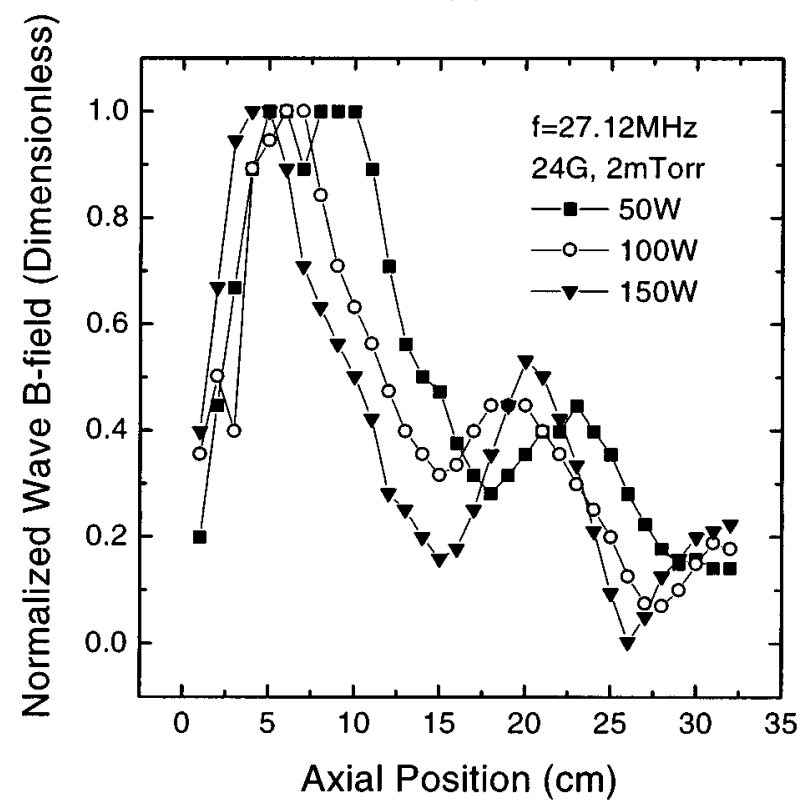

(b)

FIG. 7. (a) Beat wave patterns of the second harmonic at $\omega_{c} / \omega \sim 1.65$. The wavelengths change with the input power proportional to the plasma density. (b) Standing wave patterns at $\omega_{c} / \omega \sim 2.5$ are not changed with the input power proportional to the plasma density $(50 \mathrm{~W}: n \sim 9.0$ $\times 10^{10} \mathrm{~cm}^{-3}, 100 \mathrm{~W}: n \sim 1.8 \times 10^{11} \mathrm{~cm}^{-3}, 150 \mathrm{~W}: n \sim 2.2 \times 10^{11} \mathrm{~cm}^{-3}$ ).

not specify $k$ and $T$ as constants, but the $B$ field and the density does. The transverse wave number, $T$, can be determined by the wave field structure as given in Eqs. (13) and (14). As seen in Fig. 8, the wave amplitudes are maximum at the center regardless of wave frequency and are described by Bessel functions of the first kind of order zero. Hence the wave mode number, $m$, is equal to zero. To determine $T$ by Eq. (13) the plasma radius has to be obtained from experimental data. The plasma radius is defined by the radial position at which the wave $B$ field is zero. In this experiment the minimum value point of the wave $B$ field is regarded as the plasma radius. In the case of the second harmonic, there is no

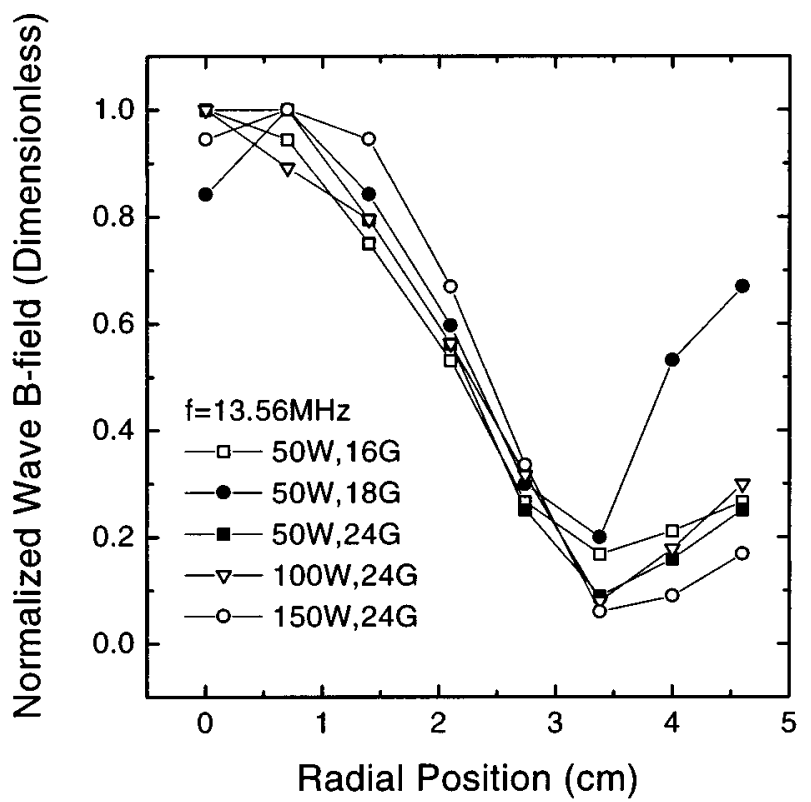

(a)

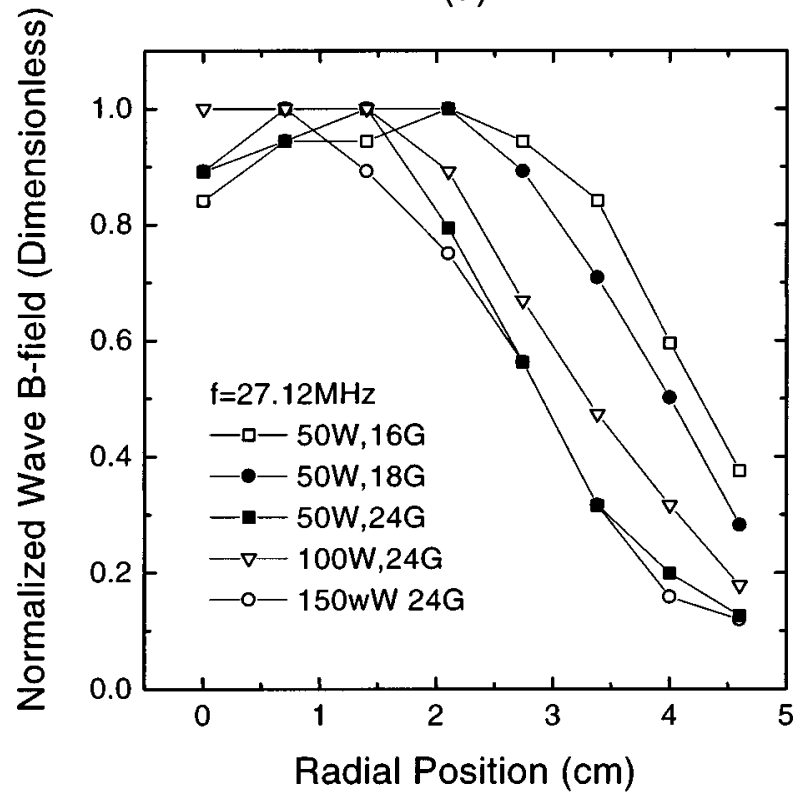

(b)

FIG. 8. Radial variations of wave $B$ field correspond to the $m=0$ mode regardless of density, $B$ field, and wave frequency. (a) Radial variations of wave $B$ field at the first harmonic. The radial position of the minimum point is taken to be the plasma radius. (b) Radial variation of wave $B$ field at the second harmonic. The chamber radius is taken to be the plasma radius.

minimum value point in the plasma and so the chamber size is considered the same as the plasma radius. The minimum value points of the wave $B$ field, in the case of the first harmonic, are a function of the $B$ field and the density. The transverse wave number at $f=13.56 \mathrm{MHz}$ is given in Fig. 9 as a function of the $B$ field and the density. At $f=27.12$ $\mathrm{MHz}, T$ is taken to be constant $T=2.405 / R$, where $R$ denotes the chamber radius.

The axially averaged density and fitted transverse wave number is used for the calculation of the dispersion relation. Waves with the first harmonic are identified as pseudosurface waves at low density $\left(n \sim 9.0 \times 10^{10} \mathrm{~cm}^{-3}\right)$ and high $B$ field 


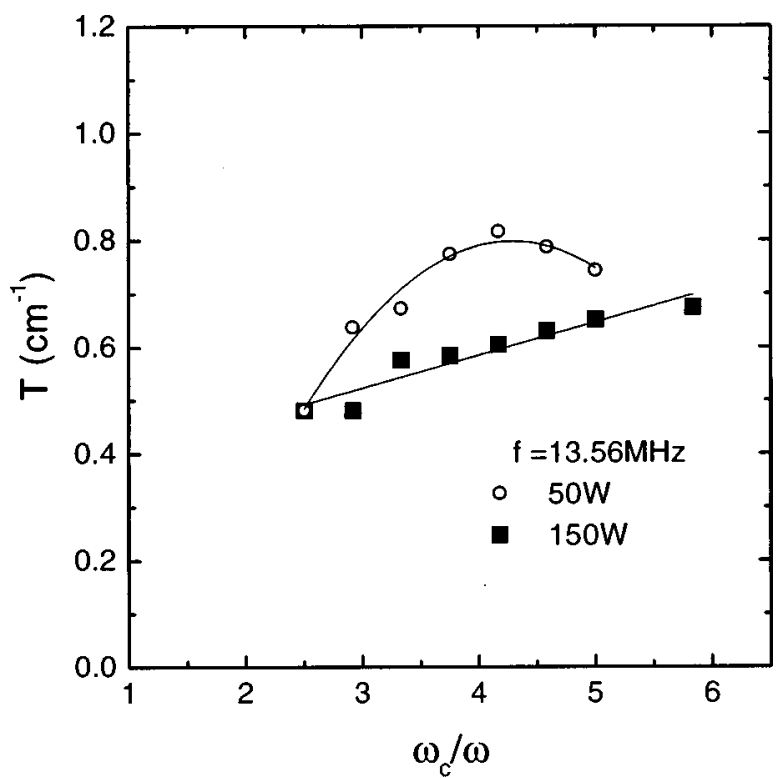

FIG. 9. The transverse wave number of the first harmonic with density and $B$ field. A least-squares fitted curve is used for calculation of the dispersion relation. In the case of the second harmonic, the transverse wave number is treated as a constant, $T=2.405 / R$, where $R$ is the chamber radius.

$\left(\omega_{c} / \omega>2.5\right)$, while waves at the second harmonic behave as helicon waves at low $B$ field $\left(\omega_{c} / \omega<2.5\right)$ as shown in Fig. 10(a). The measured helicon wave dispersion relation deviated somewhat from the experimental data, but this may be the result of the uncertainty in the density data and the uniform plasma assumption. Waves with the first harmonic propagate as helicon waves at high density $(n>2.0$ $\left.\times 10^{11} \mathrm{~cm}^{-3}\right)$ and high $B$ field $\left(\omega_{c} / \omega>3.0\right)$ as seen in Fig. 10 (b), otherwise, waves at the second harmonic propagate as pseudosurface waves at low $B$ field $\left(\omega_{c} / \omega<3.0\right)$. As theoretically expected, the pseudosurface wave at the first harmonic becomes a helicon wave at high density and high $B$ field. On the other hand, the wave at the second harmonic shows interesting behavior. The helicon waves become pseudosurface waves at high density and high $B$ field.

\section{CONCLUSION}

A plasma mode transition from $E$ discharge to $H$ discharge is observed by applying an external $B$ field unlike earlier experiments which employed increasing input power or pressure. When applying an external low $B$ field, it was found that both pseudosurface and helicon wave propagation can sustain the high density plasma. Once the plasma reaches the high density mode, inductive coupling becomes the dominant plasma heating mechanism. Even though the external $B$ field is removed from the system, plasma can be sustained by the inductively coupled discharge mode ( $H$ mode). This $H$ mode is characterized by a localized high density and luminous discharge. A wave propagation mode change by an external low $B$ field and plasma density change is observed. The azimuthally symmetric $(m=0$ mode) pseudosurface wave at the first harmonic at low density $(n \sim 9.0$ $\left.\times 10^{10} \mathrm{~cm}^{-3}\right)$ becomes a helicon wave at high density $(n$ $\left.\sim 2.2 \times 10^{11} \mathrm{~cm}^{-3}\right)$ and high $B$ field $\left(\omega_{c} / \omega>3.0\right)$, while the

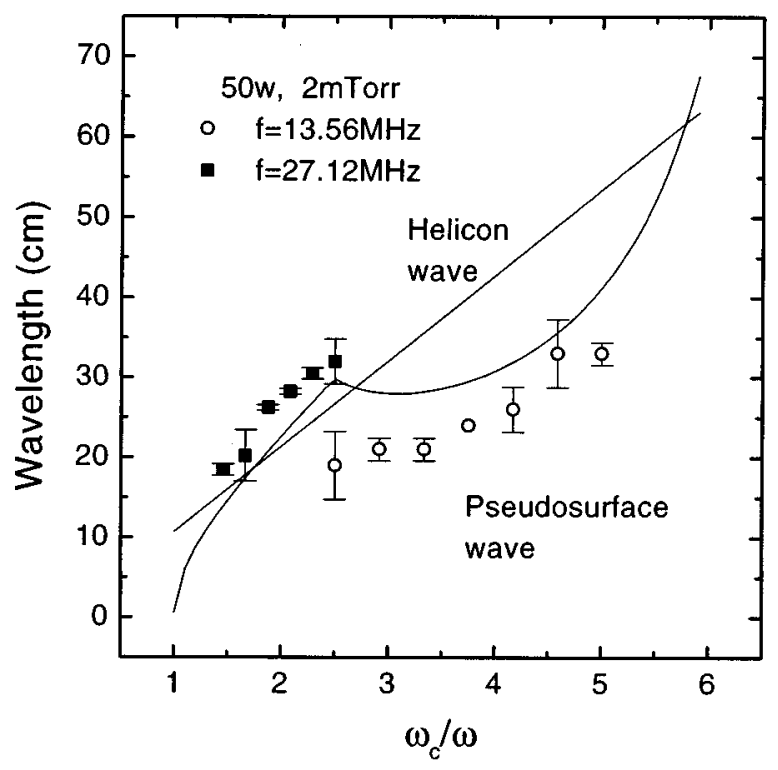

(a)

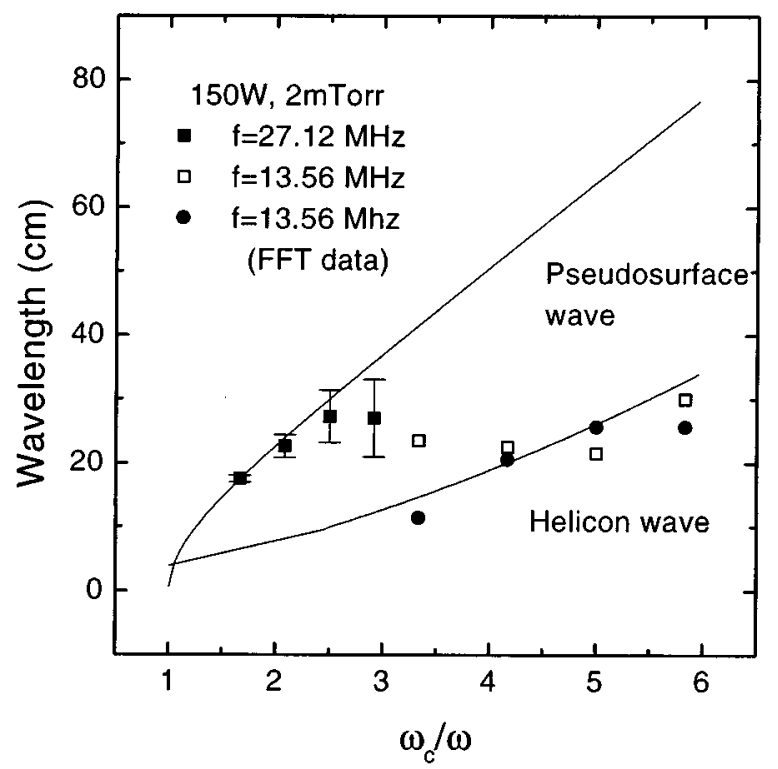

(b)

FIG. 10. Comparison of the wave dispersion relation to experimental data. (a) Waves of the first harmonic follow the pseudosurface wave dispersion relation, while waves of the second harmonic follow the helicon wave. The deviation of experimental data from the helicon dispersion relation might come from the uncertainty of the density measurement. (b) Waves of the first harmonic follow the helicon waves dispersion relation, while waves of the second harmonic follow the pseudosurface wave. Fast Fourier transformed data show good agreement with the helicon dispersion relation.

wave at the second harmonic at low density $(n \sim 9.0$ $\times 10^{10} \mathrm{~cm}^{-3}$ ) is identified as a helicon wave and changes to a pseudosurface wave at high density $\left(n \sim 2.2 \times 10^{11} \mathrm{~cm}^{-3}\right)$ and high $B$ field $\left(\omega_{c} / \omega>3.0\right)$.

\section{ACKNOWLEDGMENTS}

This research was supported by the National Science Foundation of the USA, Grant Nos. NSF EEC-8721545 and 
NSF ECS-9529565. The authors gratefully acknowledge the helpful advice of Professor J. Margot of the University of Montreal.

${ }^{1}$ H. U. Eckert, High. Temp. Sci. 6, 99 (1974).

${ }^{2}$ J. Hopwood, Plasma Sources Sci. Technol. 1, 109 (1992).

${ }^{3}$ K. A. MacKinnon, Philos. Mag. 8, 605 (1929).

${ }^{4}$ J. Hopwood, C. R. Guarnieri, S. J. Whitehair, and J. J. Cuomo, J. Vac. Sci. Technol. A 11, 147 (1993).

${ }^{5}$ I. D. Sudit and F. F. Chen, Plasma Sources Sci. Technol. 5, 43 (1996).

${ }^{6}$ L. D. Smullin and P. Chorney, Proc. IRE 46, 360 (1958).
${ }^{7}$ A. W. Trivelpiece and R. Gould, J. Appl. Phys. 30, 1784 (1959).

${ }^{8}$ I. Zhelyazkov, E. Benova, and V. Atanassov, J. Appl. Phys. 62, 2713 (1987).

${ }^{9}$ J. Margot and M. Moisan, in Plasma Processing of Semiconductor, edited by P. F. Williams (Kluwer, Dordrecht, 1997), pp. 187-210.

${ }^{10}$ E. Benova, I. Zhelyazkov, P. Staikov, and F. Cap, Phys. Rev. A 44, 2625 (1991).

${ }^{11}$ J. P. Klozenberg, B. McNamara, and P. C. Thonemann, J. Fluid Mech. 21, 545 (1965).

${ }^{12}$ F. F. Chen, Phys. Plasmas 3, 1783 (1996).

${ }^{13}$ I. D. Sudit and F. F. Chen, Plasma Sources Sci. Technol. 3, 162 (1994).

${ }^{14}$ F. F. Chen, J. Vac. Sci. Technol. A 10, 1389 (1992). 